\title{
Healing process after total cricoidectomy and laryngotracheal reconstruction: Endoscopic and histologic evaluation in a canine model
}

\author{
Kazumichi Yamamoto, MD, ${ }^{\mathrm{a}}$ Michitaka Honda, MD, ${ }^{\mathrm{a}}$ Tetsurou Yamamoto, MD, ${ }^{\mathrm{b}}$ and \\ Tatsuo Nakamura, MD, $\mathrm{PhD}^{\mathrm{a}}$
}

\begin{abstract}
Objective: The surgical procedure for subglottic stenosis is technically challenging when the vocal cords are involved and concomitant management for glottic involvement is required. After total cricoidectomy and laryngotracheal anastomosis, T-tube placement for 3 to 6 months is recommended. Bone grafts might shorten this period. We report the histologic and endoscopic changes after total cricoidectomy with or without bone grafts in a canine model to suggest an appropriate period for T-tube placement and the necessity for bone grafts.
\end{abstract}

Methods: Ten dogs underwent total cricoidectomy and laryngotracheal anastomosis with or without bone grafts harvested from the ribs. Endoscopic examination was performed monthly, and $1 \mathrm{dog}$ from both groups was humanely killed at 1, 2, 3, 6, and 12 months. The T-tube was removed before death in the dogs killed at 1,2, and 3 months and at 3 and 6 months in those killed at 6 and 12 months, respectively.

Results: Endoscopically, the glottic opening was in good condition in all dogs, except for 1 that had glottic stenosis. Histologically, active lymphocyte infiltration was observed in dense collagen fibers at the anastomosis at 1 month. At 2 and 3 months, fibroblasts were evident, suggesting active collagen fiber production. At 6 and 12 months, the collagen fibers had become looser. The bone grafts were intact and did not influence the surrounding tissue.

Conclusions: In the canine model, 6 months of T-tube placement is probably sufficient; however, 3 months of placement might not be. Additionally, no difference was found between the dogs with and without a bone graft. (J Thorac Cardiovasc Surg 2013;145:847-53)

Because of the complex structure and function of the larynx, the surgical procedure for subglottic stenosis is technically more challenging than that for tracheal stenosis, which is best treated by simple end-to-end anastomosis. Partial resection of the cricoid and subperichondrial resection of the laryngeal mucosa followed by end-to-end laryngotracheal anastomosis is currently the procedure of choice. ${ }^{1-3}$ However, when the subglottic stenosis involves the vocal cords, neither simple laryngotracheal resection nor anastomosis is possible, and concomitant management for glottic involvement is required.

\footnotetext{
From the Department of Bioartificial Organs, ${ }^{\text {a }}$ Institute for Frontier Medical Sciences, Kyoto University, Kyoto, Japan; and Department of Pathology, ${ }^{b}$ National Hospital Organization, Kyoto Medical Center, Kyoto, Japan.

Disclosures: Authors have nothing to disclose with regard to commercial support.

Dr K. Yamamoto present's address is Service of Otorhinolaryngology, Centre Hospitalier Universitaire Vaudois, Lausanne, Switzerland, Rue de Bugnon 21, Lausanne CH 1011, Switzerland.

Received for publication Nov 5, 2011; revisions received Jan 31, 2012; accepted for publication March 16, 2012; available ahead of print April 16, 2012.

Address for reprints: Kazumichi Yamamoto, MD, Department of Bioartificial Organs, Institute for Frontier Medical Science, Kyoto University, 53 Kawahara choSakyoku, Kyoto 606-8507, Japan (E-mail: granada@d3.dion.ne.jp).

$0022-5223 / \$ 36.00$

Copyright (C) 2013 by The American Association for Thoracic Surgery

doi:10.1016/j.jtcvs.2012.03.041
}

In 1992, Couraud and colleagues ${ }^{4}$ reported on total cricoidectomy followed by laryngotracheal anastomosis for subglottic stenosis with inflammatory destruction of the cricoid cartilage. In that report, 7 patients with subglottic stenosis with glottic involvement and inflammatory cricoiditis underwent the above-mentioned surgical procedure; 6 of whom were successfully decannulated postoperatively. In this technique, a T-tube is placed as a mold for the vocal cords because the cricoarytenoid joints are lost as a result of the total cricoidectomy. Couraud and colleagues ${ }^{4}$ stated that the T-tube should be placed for 3 to 6 months as a mold until the supporting tissue regains rigidity to prevent the arytenoid cartilage from falling and the vocal cords from collapsing and that it is possible that inserting a periostial free graft might shorten this period. However, these findings were determined by the clinical experience of Couraud and colleagues, ${ }^{4}$ and no investigation into this issue has been made.

The purposes of the present study were to determine the histologic and endoscopic changes after total cricoidectomy with or without bone grafting in a canine model, suggest an appropriate period for the placement of T-tube as a mold, and determine the necessity of placing a periostial free graft after total cricoidectomy. 


\section{METHODS}

\section{Animal Experiments}

Ten beagle dogs weighing $9 \mathrm{~kg}$ to $12 \mathrm{~kg}$ were randomly divided into 2 groups. In group 1, no bone graft was inserted after subperichondrial total cricoidectomy and laryngotracheal anastomosis. In group 2, a free bone graft harvested from a rib $(2 \times 1 \mathrm{~cm})$ was fixed on the perichondrium and left in place after subperichondrial total cricoidectomy followed by laryngotracheal anastomosis.

The detailed surgical techniques have been described in a previous human case report ${ }^{5}$; almost the same procedure was used in the present study (Figure 1). The dogs were anesthetized with intramuscular ketamine hydrochloride $(15 \mathrm{mg} / \mathrm{kg})$ and xylazine hydrochloride $(3 \mathrm{mg} / \mathrm{kg})$, and anesthesia was maintained with halothane inhalation. A horizontal cervical incision was made at the level of the lower border of the cricoid cartilage. The trachea was opened between the second and third tracheal rings to prepare a flap of the membranous portion of the first and second cartilage levels, and cross-field ventilation was started intraoperatively. After subperichondrial resection of the anterior arch of the cricoid cartilage, a laryngofissure was performed to observe the intraluminal airway. Subperichondrial total cricoidectomy was performed, leaving the exterior perichondrium attached to the esophagus. Next, the intraluminal mucosa was cut along the lower border of the vocal cords. The flap of the membranous portion of the trachea was sutured to the posterior commissure of the larynx to cover the perichondrium with or without a free bone graft from the rib (taken from the lowest palpable rib), and an end-to-end laryngotracheal anastomosis was performed with 3-0 Vicryl (Ethicon EndoSurgery, Cincinnati, Ohio) interrupted sutures. In both groups, a T-tube was placed before the anastomosis as a mold for the vocal cords, with the upper edge of the T-tube positioned above the opening of the vocal cords to ensure the cords were kept open. The upper end of the T-tube is capped when the procedure is performed in humans; however, capping was not performed for the dogs because of the decreased aspiration in the quadrupedal posture.

A 500-mg dose of ampicillin was injected intramuscularly on the day of surgery and on the first and second postoperative days. No additional care other than routine management was provided for the dogs.

\section{Endoscopic Examination}

Endoscopic examinations were performed monthly with the dogs under general anesthesia with intramuscular ketamine hydrochloride $(10 \mathrm{mg} / \mathrm{kg})$ and xylazine hydrochloride $(4 \mathrm{mg} / \mathrm{kg}$ ) to observe the condition of the vocal cords and T-tube. Dogs in both groups were humanely killed by an overdose of injected sodium pentobarbital at $1,2,3,6$, or 12 months postoperatively. The T-tube was removed just before death in the dogs killed at 1,2, or 3 months postoperatively, and the conditions around the vocal cords and subglottic space were observed. In the dogs killed at 6 and 12 months postoperatively, the T-tube was removed 3 and 6 months after the operation, respectively, and endoscopic examination was performed monthly.

\section{Histologic Examination}

Each dog in both groups was humanely killed as described in the previous section. En bloc resection of the reconstructed larynx and the upper third of the trachea and esophagus was performed for histopathologic examination. After the specimens had been fixed in formalin for 48 hours, each lesion was sliced at 4-mm intervals, and paraffin blocks were prepared. Sections of each block were then prepared, stained, and examined. The sections were stained with hematoxylin and eosin to evaluate the infiltration of inflammatory and mesenchymal cells into the surrounding tissue around the anastomosis and with Masson's trichrome stain to evaluate the proliferation and maturation of the collagen bundles around the perichondrium left after the subperichondrial cricoidectomy. The histologic findings were evaluated according to the type and degree of infiltration of the inflammatory cells and the maturation of the collagen bundles at each interval. The differences in the findings for the 2 groups with or without a bone graft were also evaluated.

The Animal Experiment Committee, Kyoto University, approved the present study, and all the surgical and euthanasia procedures were performed in accordance with the guidelines of this committee.

\section{RESULTS}

All procedures were performed successfully in all study animals. All the dogs survived until death, and no complications such as anastomotic dehiscence or stenosis occurred, except for 1 case of posterior glottic stenosis evolving after decannulation of the T-tube. Details of the cases are shown in Table 1. During the study period, respiratory complications, such as tube obstruction from bronchial secretions or pneumonia, that required aspiration bronchoscopy or additional antibiotic therapy were not observed.

\section{Endoscopic Findings}

In both groups, in the dogs killed at 1, 2, and 3 months postoperatively (Figure 2), no abnormal findings were observed endoscopically, and the glottic opening was in good condition after the T-tube was removed just before death.

In group 1, in the dog killed at 6 months postoperatively, the glottic opening was in good condition just after the T-tube was removed at 3 months after the operation. At 6 months postoperatively, the glottic opening was still in good condition, although the anterior thirds of the vocal cords were in contact, although not fused with each other. This dog had a breathy voice when barking without stridor at rest because the vocal cords were fixed in the open position. In group 2, in the dog killed at 6 months postoperatively, the glottic opening was in good condition just after the T-tube was removed 3 months after the operation. However, posterior glottic stenosis developed 2 weeks after the T-tube was removed. Finally, total posterior glottic closure was observed, and tracheostomy was required to maintain respiration. This dog exhibited stridor at rest before the tracheostomy was placed again because of the posterior glottic stenosis.

In group 1, in the dog killed at 12 months postoperatively, the glottic opening was in good condition just after the T-tube was removed at 6 months after the operation. At 12 months after the operation, the glottic opening was sufficient for respiration; however, the anterior half of the vocal cords were in contact but not fused with each other. In the dog in group 2, killed 12 months postoperatively, the glottic opening was in good condition just after the T-tube was removed at 6 months after the operation. At 12 months after the operation, the glottic opening was in good condition. However, the anterior thirds of the vocal cords were in contact but not fused with each other, with slight granulation on the left arytenoid cartilage. These $2 \operatorname{dogs}$ had a breathy 

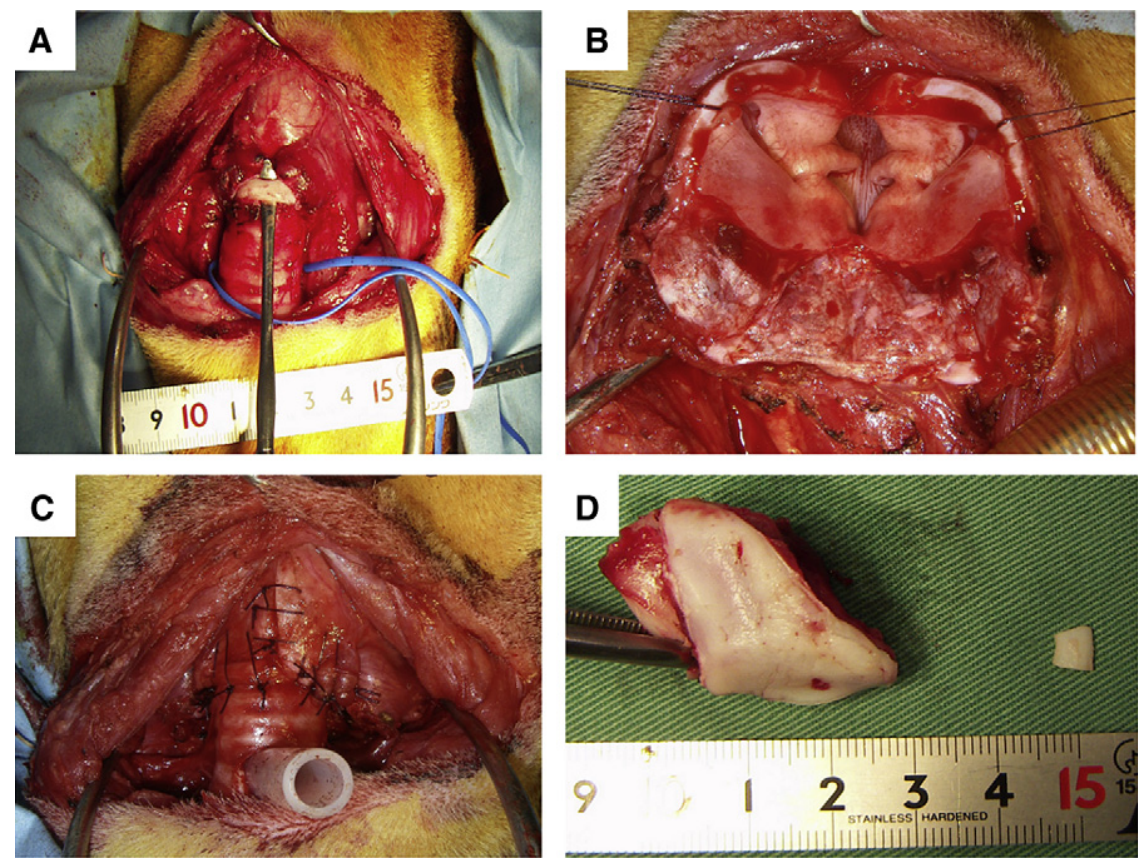

FIGURE 1. Surgical procedures. A, Subperichondrial resection of the anterior arch of the cricoid cartilage. B, Subperichondrial total cricoidectomy and resection of the intraluminal mucosa. Care must be taken to leave the exterior pericondrium attached to the esophagus. The intraluminal mucosa is cut along the lower border of the vocal cords. C, End-to-end laryngotracheal anastomosis is performed with interrupted 3-0 Vicryl sutures. The T-tube is placed in front of the anastomosis as a mold for the vocal cords, and the upper edge of T-tube is positioned above the opening of the vocal cords to keep them open. $\mathrm{D}$, Resected cricoid cartilage (left), and resected portion of the anterior arch of the cricoid cartilage.

voice when barking without stridor at rest because the vocal cords were fixed in the open position.

\section{Histologic Findings}

Observations on the histologic specimens at the anastomosis between the membranous portion of the trachea and interarytenoidal portion 1, 2, 3, 6, and 12 months after the operation in both groups are described below (Figure 3).

In group 1 (no bone graft), 1 month after the operation, active infiltration of lymphocytes and a few plasma cells were observed in the dense collagen fibers between the membranous portion of the trachea and the muscle layer of the esophagus. At 2 and 3 months after the operation, lymphocyte infiltration was reduced, and fibroblasts were evident within the dense collagen fibers, suggesting active collagen fiber production. At 6 and 12 months postoperatively, fibroblasts were less evident and the collagen fibers at the anastomosis had become looser than at 2 and 3 months postoperatively. In addition, no inflammatory reaction was present at this point.

TABLE 1. Details of the cases

\begin{tabular}{lcccl}
\hline Case no. & $\begin{array}{c}\text { Interval to } \\
\text { decannulation }(\mathbf{m})\end{array}$ & $\begin{array}{c}\text { Interval to } \\
\text { death }(\mathbf{m})\end{array}$ & $\begin{array}{c}\text { Endoscopic findings } \\
\text { after removal of T-tube }\end{array}$ & \multicolumn{1}{c}{ Histologic findings } \\
\hline Group 1 (no graft) & 1 & & - & Active infiltration of lymphocytes \\
A & 2 & 1 & - & Dense collagen fibers \\
B & 3 & 2 & - & Dense collagen fibers \\
C & 3 & 6 & Opening: good, no granulation & Loose collagen fibers \\
D & 6 & 12 & Opening: good, no granulation & Loose collagen fibers \\
E & 1 & 1 & - & Active infiltration of lymphocytes \\
Group 2 (bone graft) & 2 & 2 & - & Dense collagen fibers \\
F & 3 & 3 & - & Dense collagen fibers \\
G & 3 & 6 & Posterior glottic stenosis & Loose collagen fibers \\
H & & & & - \\
I & 6 & 12 & Opening: good, slight granulation & Loose collagen fibers \\
J & & & & - \\
\hline
\end{tabular}



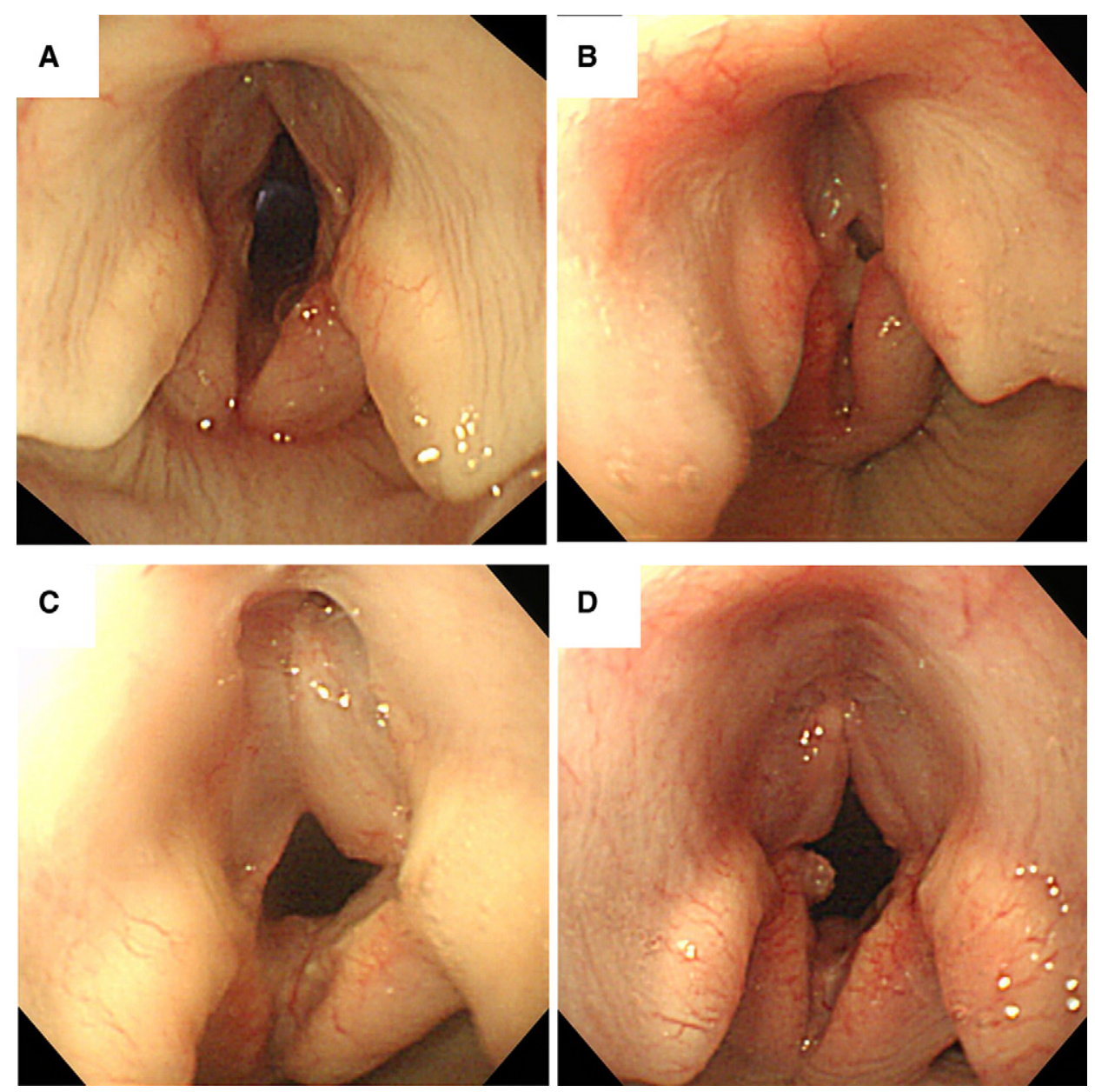

FIGURE 2. Endoscopic findings 3 months after the removal of the T-tube. The T-tube was placed for (A) 3 months without a bone graft, (B) 3 months with a bone graft, (C) 6 months without a bone graft, and (D) 6 months with a bone graft.

In group 2 (bone graft), 1 month after the operation, active infiltration of neutrophils was observed around the Vicryl sutures used to fix the bone graft. Active infiltration of lymphocytes and a few plasma cells was also observed in the dense collagen fibers; there was no apparent difference compared with group 1 . At 2 and 3 months, lymphocyte infiltration was reduced and fibroblasts were evident within the dense collagen fibers, suggesting active collagen fiber production. Also, no apparent difference was found compared with group 1 . Six months after the operation, epithelial cells around the anastomosis were infiltrated by inflammatory cells such as neutrophils. This finding was compatible with the posterior glottic stenosis observed endoscopically. Beneath the epithelial layer, fibroblasts were less evident and collagen fibers at the anastomosis had become looser than at 2 and 3 months after the operation. At 12 months postoperatively, the fibroblasts were less evident and the collagen fibers at the anastomosis had become looser than at 2 and 3 months after the operation. No inflammatory reaction was present at this point. Throughout the study period, the bone grafts fixed in place were intact; they were not absorbed and did not influence the surrounding tissue.

\section{DISCUSSION}

Acquired subglottic stenosis is a complex condition, and its treatment is still controversial. A recent meta-analysis indicates that laryngotracheal anastomosis is the procedure of choice for suitable patients and provides better outcomes than other procedures. ${ }^{6}$ However, subglottic stenosis with glottic involvement is particularly more complex than without glottic involvement; its treatment has not been established.

Laryngotracheal resection and anastomosis were first developed in the 1970s and 1980s by Pearson and colleagues, ${ }^{7}$ Grillo, ${ }^{8}$ and Couraud and colleagues ${ }^{9}$ for subglottic stenosis, which had previously been treated by laryngoplasty - with or without a bone or cartilage graft. This type of resection of the cricoid ring that is performed together with resection 

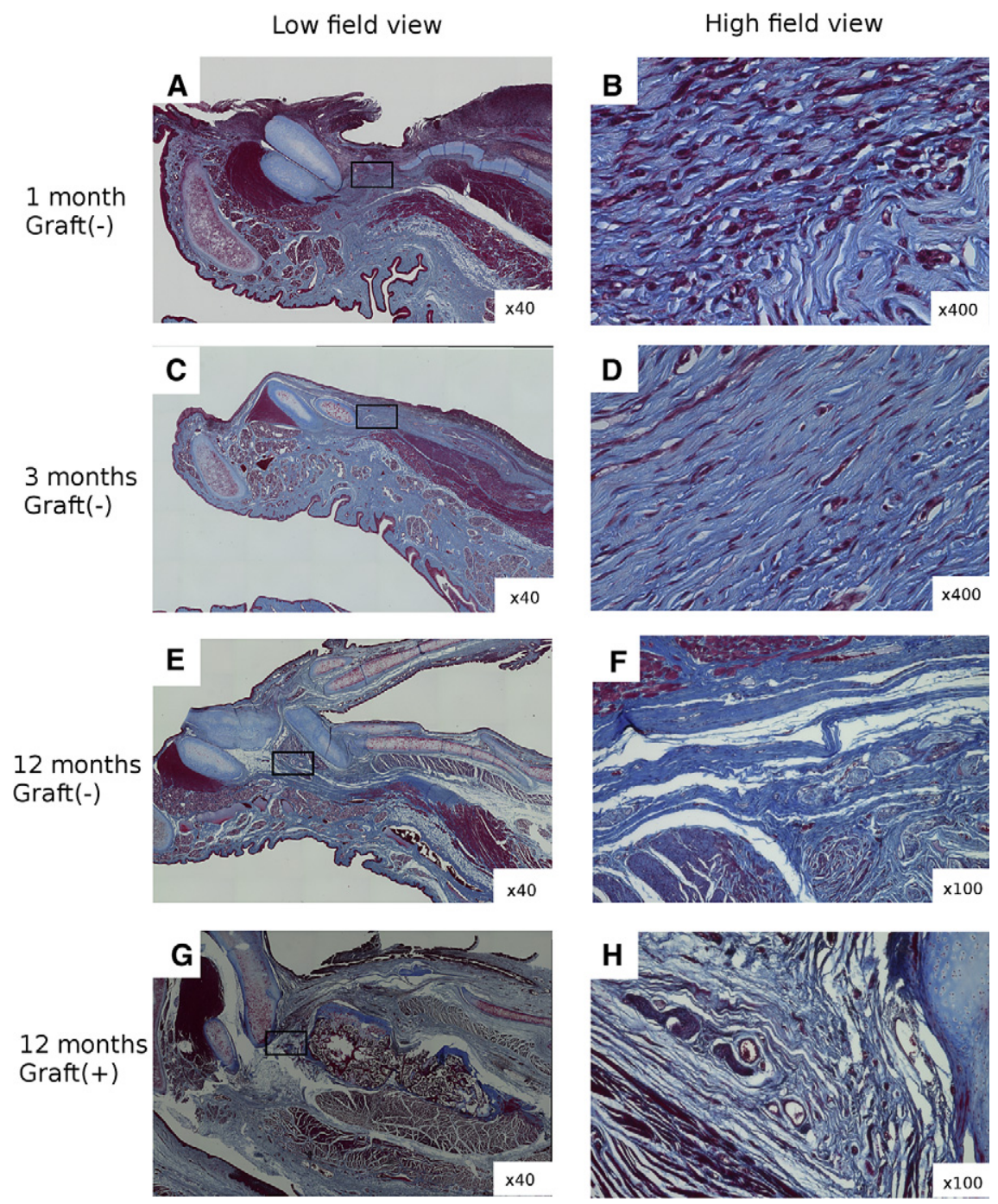

FIGURE 3. Histologic findings from Masson's trichrome staining. At 1 month after the operation, active infiltration of lymphocytes and a few plasma cells was observed in the dense collagen fibers between the membranous portion of the trachea and the muscle layer of the esophagus: (A) $\times 40$ and (B) $\times 400$ magnification. At 3 months after the operation, lymphocyte infiltration was reduced and fibroblasts were evident within the dense collagen fibers, suggesting active collagen fiber production: (C) $\times 40$ and (D) $\times 400$ magnification. At 12 months after the operation, fibroblasts were less evident and collagen fibers at the anastomosis had become looser than at 3 months after the operation; there was no inflammatory reaction at this point: (E) $\times 40$ and (F) $\times 100$ magnification. Throughout the study period, the bone grafts fixed in place were intact and were not absorbed nor did they influence the surrounding tissue: $(\mathrm{G}) \times 40$ and $(\mathrm{H}) \times 100$ magnification.

of the affected mucosa and is followed by laryngotracheal anastomosis is highly effective for subglottic stenosis without glottic involvement. However, this technique is insufficient for subglottic stenosis with glottic involvement, and concomitant management of the vocal cords is required.

In 1992, Maddaus and colleagues ${ }^{3}$ reported laryngotracheal reconstruction with laryngofissure for subglottic stenosis with glottic involvement by scar formation at the intraarytenoidal mucosa. With this technique, the intraarytenoidal scar is resected by laryngofissure followed by laryngotracheal anastomosis with T-tube placement for several weeks or months to prevent the vocal cords from closing just after the operation. This technique is useful for subglottic stenosis with glottic involvement by intraarytenoidal scar formation. However, if the inflammatory damage to the cricoid cartilage exists together with the intra-arytenoidal scar, only resecting the scar is insufficient for opening the vocal cords owing to the presence of ankylosis of the cricoarytenoid joint. George and colleagues ${ }^{10}$ reported a high success rate for laryngotracheal resection and anastomosis performed together with posterior splitting of the cricoid body for pediatric patients with such complex conditions. This technique is especially useful for subglottic stenosis with closed vocal cords owing to 
ankylosis of the cricoarytenoid joint. However, in most cases, acquired subglottic stenosis is caused by long-term intubation or high tracheostomy; therefore, total destruction of the posterior cricoid plate caused by cricoiditis or cartilage necrosis is sometimes observed. Couraud and colleagues ${ }^{4}$ reported 7 cases in which patients with this severe condition underwent total cricoidectomy followed by laryngotracheal anastomosis through temporal laryngofissure; in 6 patients, decannulation was successfully performed. However, this technique is highly demanding, and no other report of this method has been published. Couraud and colleagues ${ }^{4}$ stated that the T-tube should be placed for 3 to 6 months as a mold until the supporting tissue regains the rigidity to prevent the arytenoid cartilage from falling and collapsing the vocal cords and that it is possible that placing a periostial free graft would shorten that period. However, these statements were determined from their clinical experience, and no investigation into these issues has been performed.

In the present study, we observed the endoscopic and histologic changes after total cricoidectomy with or without a bone graft in a canine model to suggest an appropriate period for the placement of a T-tube as a mold and the necessity of placing a periostial free graft after total cricoidectomy. From the histologic findings, the collagen fibers around the anastomosis 3 months after the operation were still immature compared with those 6 months after the operation, although the acute and chronic inflammatory changes had almost disappeared at 3 months. This might suggest that scar contraction around the anastomosis can occur until collagen fibers become sufficiently loose and stable. In the dogs in group 2, the glottic opening was in good condition just after the T-tube was removed 3 months after the operation; however, posterior glottic stenosis evolved 2 weeks after the T-tube was removed. Couraud and colleagues ${ }^{4}$ reported that after the T-tube was removed at 3 months postoperatively. However, a T-tube was placed again for another 3 months because of residual laryngeal collapse in 2 of the 7 patients who underwent total cricoidectomy. This finding might be compatible with our results. Furthermore, they reported that they placed a periosteal or a perichondrial graft to accelerate the recovery of rigidity after perichondrial total cricoidectomy. However, in our study, the grafts left in place did not have any positive effects on the surrounding areas nor were any grafts absorbed during the study period. From these results, we assume that 6 months of T-tube placement is probably sufficient; however, 3 months of placement might not be sufficient because the collagen fibers are still immature at 3 months. In addition, we found no apparent difference between those with bone graft and those without at 1 year and the placement of the periostial free graft on the outer perichondrium after the total cricoidectomy seems to have no additional effects on the healing process around the anastomosis. Our conclusions are limited by the small sample size and short observation period. Additional studies are required to obtain a better understanding.

The present study had several important limitations. First, because this was an animal study, the results cannot be directly applied to humans. However, the postoperative histologic changes after laryngotracheal reconstruction have not been previously described, especially for laryngeal stenosis involving the vocal cords. There has been no evidence to define the optimal period of T-tube placement or the necessity for a cartilage graft; therefore, treatment decisions have relied on personal experience. We used a canine model because the dog larynx is similar to that of humans with respect to the size, style, and function. In this sense, the present study has several implications for the adequate treatment of this complex condition.

Second, the sample size was very small, with only $1 \mathrm{dog}$ per condition, and no control group was used. Because this was not a comparative study, but a descriptive study after a surgical procedure, it is difficult to draw definitive conclusions. In addition, the present animal study used a large animal model, severely limiting the number of animals. Therefore, we only examined $1 \mathrm{dog}$ per condition to study the correlations. A larger number of study animals would be required to derive more robust conclusions.

Third, the surgical procedure was performed in normal dogs without subglottic stenosis or glottic involvement. Subglottic stenosis is mostly caused by inflammatory changes at the larynx, and a disease model is generally required for this type of investigation. However, the concept of laryngotracheal resection and anastomosis for inflammatory subglottic stenosis is "resection of all affected lesions" and "anastomosis of the healthy mucosa and cartilage." In this sense, we dared not develop an animal disease model for the present study. There might have been an influence of inflammation around the anastomosis; however, compared with laryngofissure and laryngeal enlargement or endoscopic laser therapy, in which the affected portion is left in place, the influence of inflammatory changes might be much less significant. Despite these limitations, these results provide many suggestions for clinical situations, because this type of procedure is not widely used and little information is available.

\section{CONCLUSIONS}

In a canine model, 6 months of T-tube placement is probably sufficient; however, 3 months of placement might not be because the collagen fibers at 3 months were still immature. In addition, we found no apparent difference at 1 year between those with and without a bone graft. These results suggest several clinical implications for human cases.

We would like to thank Drs Masato Uji, Fumitsugu Kojima, Akira Nakata, and Yuji Nishizawa for their technical assistance. 


\section{References}

1. Grillo HC, Mathisen DJ, Wain JC. Laryngotracheal resection and reconstruction for subglottic stenosis. Ann Thorac Surg. 1992;53:54-63.

2. Pearson FG, Gullane P. Subglottic resection with primary tracheal anastomosis including synchronous laryngotracheal reconstruction. Acta Otorhinolaryngol Belg. 1995;49:389-96.

3. Maddaus MA, Toth JL, Gullane PJ, Pearson FG. Subglottic tracheal resection and synchronous laryngeal reconstruction. J Thorac Cardiovasc Surg. 1992; 104:1443-50.

4. Couraud L, Jougon JB, Velly JF. Surgical treatment of nontumoral stenoses of the upper airway. Ann Thorac Surg. 1995;60:250-9.

5. Yamamoto K, Tomiyama K, Mitsuoka M. Total cricoidectomy and laryngotracheal reconstruction for subglottic stenosis with glottic involvement. Interact Cardiovasc Thorac Surg. 2011;13:351-3.
6. Yamamoto K, Kojima F, Tomiyama K, Nakamura T, Hayashino Y. Meta-analysis of therapeutic procedures for acquired subglottic stenosis in adults. Ann Thorac Surg. 2011;91:1747-53.

7. Pearson FG, Cooper JD, Nelems JM, Van Nostrand AW. Primary tracheal anastomosis after resection of the cricoid cartilage with preservation of recurrent laryngeal nerves. J Thorac Cardiovasc Surg. 1975;70: 806-16.

8. Grillo HC. Primary reconstruction of airway after resection of subglottic laryngeal and upper tracheal stenosis. Ann Thorac Surg. 1982;33:3-18.

9. Couraud L, Brichon PY, Velly JF. The surgical treatment of inflammatory and fibrous laryngotracheal stenosis. Eur J Cardiothorac Surg. 1988;2:410-5.

10. George M, Jaquet Y, Ikonomidis C, Monnier P. Management of severe pediatric subglottic stenosis with glottic involvement. J Thorac Cardiovasc Surg. 2010; 139:411-7. 